\title{
Robotic Hair Harvesting System: A New Proposal
}

\author{
Xiang Lin, Toji Nakazawa, Ryuya Yasuda, Etsuko Kobayashi, \\ Ichiro Sakuma, and Hongen Liao \\ Graduate School of Engineering, University of Tokyo, Japan \\ shanelinnz@gmail.com, liao@bmpe.t.u-tokyo.ac.jp
}

\begin{abstract}
Follicular Unit Extraction (FUE) has become a popular hair transplanting method for solving male-pattern baldness problem. Manually harvesting hairs one by one, however, is a tedious and time-consuming job to doctors. We design an accurate hair harvesting robot with a novel and efficient end-effector which consists of one digital microscope and a punch device. The microscope is first employed to automatically localize target hairs and then guides the punch device for harvesting after shifting. The end-effector shows average bias and precision of $0.014 \mathrm{~mm}$ by virtue of a rotary guidance design for the motorized shifting mechanism.
\end{abstract}

\section{Introduction}

At least half of the male population by age 50 in this world have a hair loss problem. About 250,000 hair restoration procedures were estimated to be performed worldwide in 2008 [1]. Hair transplanting surgery is considered as the most efficient solution to this problem.

The follicular unit extraction (FUE) technique, proposed in [3, is considered as one of the most advanced methods for transplanting. It uses a small sharp punch to directly extract follicular units one by one from man's scalp to avoid leaving a linear scar. The linear scar is often observed in the currently most popular method, follicular unit transplanting (FUT) [2]. FUE has been proven less invasive, faster recovery, and less noticeable scarring. The main disadvantages are related to potentially high rates of follicle transection and the requirement of a high degree of technical skill to doctors, which prevents the technique from being widely accepted.

A robotic system can assist doctors to both improve the accuracy and reduce the variability in operation results due to an individual's technique. Little research has been done in this area in last decade. Onda et. al. claimed a robotic harvesting system in 4 but it still had to be manually held by doctors. Gildenberg proposed a real robotic system in [5], which used a stereotactic video system for $3 \mathrm{D}$ reconstruction of target hairs. The system was further implemented in [6] but unfortunately no detail report has been presented to demonstrate how accurate the system is by using the stereotactic system.

In this paper, we design a new accurate harvesting robot with a novel endeffector which consists of one digital microscope (a single camera) and a punch

G. Fichtinger, A. Martel, and T. Peters (Eds.): MICCAI 2011, Part I, LNCS 6891, pp. 113-120, 2011. (C) Springer-Verlag Berlin Heidelberg 2011 
device. The microscope is employed as the first step in the process to automatically localize target hairs with a customized image-based visual servoing method. The punch device is then shifted by a motor to the same position as the microscope for harvesting. R-guide is applied to provide the shifting mechanism which greatly improves the system accuracy and simplifies the mechanical design. In this paper we focus on the system design, particularly the end-effector design.

The remainder of the paper is organized as follows. The design of the whole system is described in Sect. 2. Section 3 gives the details of the end-effector. The calibration method for the end-effector is provided in Sect. 4. Experiments and results are presented in Sect. 5, followed by conclusions in Sect. 6.

\section{System Design}

The target of the system is to accurately extract hairs one by one from a donor area. The harvesting process of a single hair includes the following steps. Firstly, the system finds a suitable hair in the field of view and move the punch device in front of it. A sharp needle is then inserted into the skin along the hair axis and dissects the whole hair follicle from the surrounding tissues. The insertion terminates once the follicle is extracted or a fixed depth is met. For safety reasons, the system cannot be designed as fully automatic and the whole process must be under doctor's supervision.

Figure 1 shows the design of our proposed system which consists of four main parts, a robot arm, an end-effector, a computer, and a user interface. The robot arm (VP-6242E, DENSO, Japan) has six degrees of freedoms (DOFs) and is able to move to any pose with the repeatability of $0.02 \mathrm{~mm}$ within the region of interest. The end-effector includes a punch device for harvesting and a digital microscope for inspection and localization. The two devices switch about each other to complete both the localization task and the harvesting task. The user interface has three functions, monitoring the process in real time, assisting doctors to choose suitable hair candidates, and receiving commands from doctors. The computer is used for image processing, image acquisition and robot arm controlling. The process has the following two phases:

\section{Localization phase}

1. The digital microscope is shifted by a stepping motor to the working position shown in Fig. 2A. Doctors select which hair should be harvested by clicking on the image captured from the microscope.

2. An image processing program is executed to obtain the hair's orientation and position on the $2 \mathrm{D}$ image.

3. The orientation and position information is fed to a customized visual servoing software. The software calculate the necessary movement that the robot arm needs to move the hair to a target pose.

4. The movement commands are sent to the robot arm for action and the image on the screen is updated after moving. 


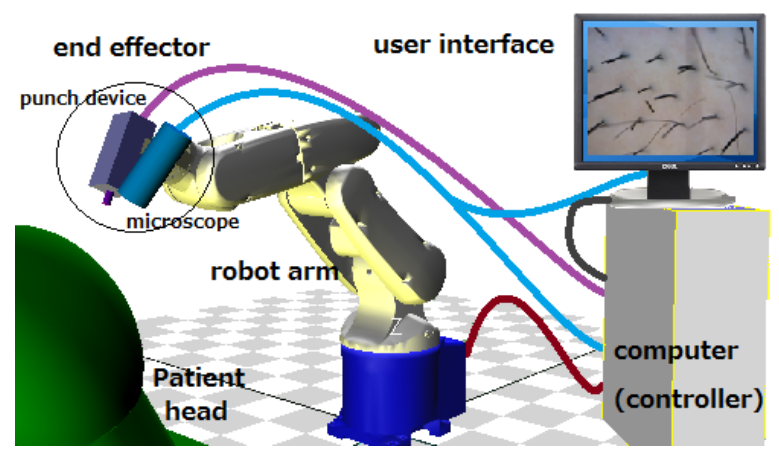

Fig. 1. Hair harvesting system, consisting of a robot arm, an end-effector, a user interface and a computer

5. The image processing program tracks the position and orientation of the hair in the updated image.

6. The robot arm stops once the hair is presented with the target pose in image. Otherwise a recursive process from Step 3 to Step 5 is executed until the hair reaches the target pose.

It should be noticed that different target poses may be available for use but a suitable pose should have a unique presentation in the image and must have enough information for the image-based visual servoing to process. We proposed of using the pose which is located on the microscope optical axis and have the same orientation as the axis. The hair should be shown as a black dot at the center of the image (Fig. $3 \mathrm{E}$ ) once the pose is reached. The distance between the microscope and the target hair can be determined by the analysis of image blurriness because the microscope with high magnification often has a very short depth of field.

\section{Harvesting phase}

1. The punch device is shifted to the working position (Fig. 2B). The needle of the punch device is advanced into the skin by a linear actuator.

2. The needle stops once the embedded sensor detects that the hair has already been dissected from the surrounding tissues or a maximal distance under the skin is reached.

3. The needle is withdrawn from the skin and the current process finishes. Doctors determine if a new process will be started by selecting another hair on the screen.

There is no visual servoing in the harvesting phase. The system is simply designed in a open-loop way without active compensation for misalignment from the shifting. The accuracy is relied on mechanical calibration which has been proven to meet the requirements in Sect. 4. 


\section{End-Effector Design}

A reliable and cost-efficient mechanical design for the shifting mechanism is critical to the system accuracy. In this paper, we use the R-guide (HCR 15A+60/ 150R, THK, Japan) for high-accurate rotary guidance in a large working area. The microscope and the punch device are installed on the R-guide through an arc-shaped rack. A small angle less than 30 degrees is set between them to reduce the shifting time. The motion of the R-guide is driven by a light weighted stepping motor (CMK223AP-SG7.2, Oriental Motor, Japan) through the gear at the rim of the rack. There are two main advantages of using a R-guide here:

1. Able to reduce system errors: The working area is designed at the center of the R-guide. Any position error caused at the rim of the rack can be reduced at the center. For example, there often exists a backlash between the pinion and the gear on the rack. If the backlash is assumed to be $1.0 \mathrm{~mm}$ and the radius of the rack is set to $160 \mathrm{~mm}$, the angular error caused by the backlash will be about 0.36 degrees. In an ideal situation, if the needle exactly passes the center and a hair is just located at the center, this angular error will cause about $0.034 \mathrm{~mm}$ position error at the tip of a $5.5 \mathrm{~mm}$ long hair (the average hair length after shaving in transplanting surgeries). This capability will also be proved by the repeatability tests in the experimental section.

2. Able to monitor needle insertion with microscope: It is necessary to monitor the depth of insertion in real time for safety purposes. One solution is to measure the needle length which is left above the skin. It can estimated from the image captured by the microscope. The requirement is that the distance between the working space and the microscope must be kept constant since the microscope has a short depth of field. This can be satisfied in this design. The insertion area above the skin can always be clearly viewed from the microscope during the whole process. Some specific marks are needed on the needle for image analysis.

The needle insertion is served by a linear actuator/motor (CCM05M-072-CD01-EN-E1, THK, Japan) which has a high resolution of $1.64 \mu \mathrm{m}$ and a relative high speed of $1.0 \mathrm{~m} / \mathrm{s}$. The actuator thrust can go up to $10.4 \mathrm{~N}$ which is enough to pierce human's scalps. It can be automatically deactuated to provide a safe stop function when the maximum thrust lasts more than several seconds. The needle has an outer diameter of $0.9 \mathrm{~mm}$ and a $0.1 \mathrm{~mm}$ thick wall. It is installed at the tip of a hollow spline shaft and controlled by the linear actuator. In order to automatic extract the follicular unit after harvesting, a vacuum system is connected to the other end of the shaft. A photoelectric sensor (not shown) will be installed on the path to the vacuum flow. It stops the linear actuator from further insertion once the hair is detected.

The microscope is attached to the rack by an adjustable bracket which can be manually adjusted in 3 DOFs for calibration. Detail adjustment will be described in Sect. 4. A high speed camera of $120 \mathrm{fps}$ and 659 x 494 resolution (Prosilica GC660, AVT, USA) is applied in this application. The lens is adjustable with the magnification from $5 \mathrm{x}$ to $50 \mathrm{x}$. 


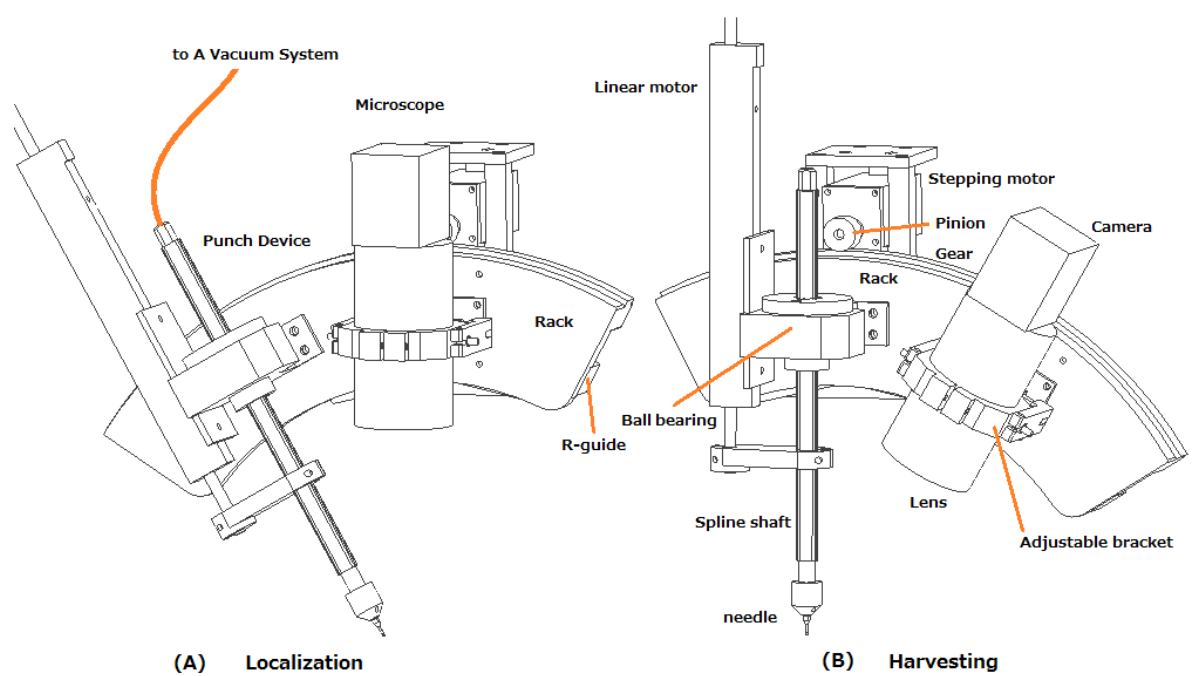

Fig. 2. Two phases, (A) localization phase when the microscope is in the working position, (B) harvesting phase when the punch device is in the working position

\section{End-Effector Calibration}

The system could provide high accuracy only if the needle insertion axis was exactly located as same as the microscope axis when they were in the working position. A new calibration method of the end-effector was proposed here for this alignment. It was actually a hair implanting process, the reverse of a normal harvesting process. The hair phantom used here was a small piece of $0.5 \mathrm{~mm}$ size pencil lead. It was implanted into a flat stage in the same orientation as the shaft axis and also on the path where the needle passed. The microscope position was then adjusted until its axis had the same direction as the pencil lead. The details are described as follows.

1. Implant the pencil lead into the stage with a specific pose: Some silicon gel was put on the stage in advance. The punch device was shifted to the working position (Fig. 3A). The pencil lead was manually inserted into the needle. It was slowly advanced to the stage (Fig. $3 \mathrm{~B}$ ) with the needle and inserted into the gel on the stage. The lead was left in the gel until the gel became dry. In this way, it could be ensured that the lead had the same orientation as the shaft and was also located on the path of the needle.

2. Adjust microscope's orientation and position: The microscope was now shifted to the working position. The lead might not be located at the image center (Fig. 3D) or even outside of the field of view. The 3 DOFs bracket (Fig. 3 $\mathrm{C}$ ), with two rotations and one translation, was used for the adjustment. The translation in the horizontal direction(green arrow) would be made up by the rotation of the stepping motor. It should be noticed that Rotation $\mathrm{A}$ and Translation $\mathrm{C}$ must be adjusted together since both of them would change 

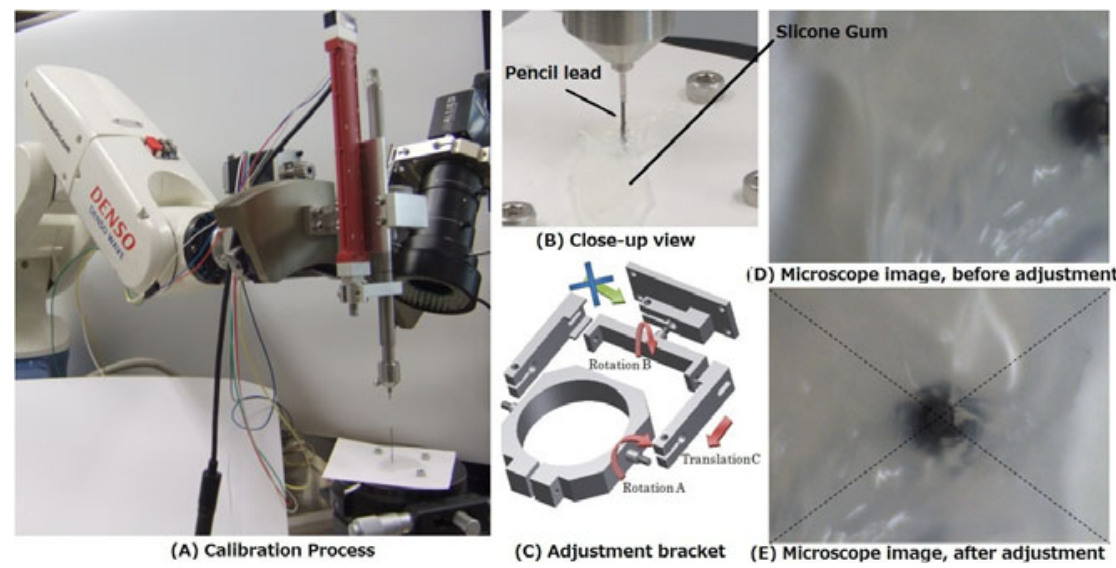

Fig. 3. Calibration process. (A) the stage and two steps in the process, (B) a close-up view of the fixing the pencil lead by silicone gel, (C) 3 DOFs adjustable bracket, (D) before adjustment, and (E) shown as a black dot at the center after adjustment

the vertical position, while Rotation A would change the orientation as well. After adjustment the lead should be shown at the image center (Fig. $3 \mathrm{E}$ ).

\section{$5 \quad$ Experiments and Results}

All the evaluations were performed on 2D images obtained from the microscope. Microscope pose repeatability, position errors and orientation errors were evaluated. A piece of paper with a number of $3.0 \mathrm{~mm}$ size black circles was fixed on the adjustable stage which had been used in the calibration step (Fig. 4A). The circle centers were clearly marked with crosses in Fig. $4 \mathrm{D}$.

Pose Repeatability: An image such as Fig. 4D was captured from the microscope. The repeatability was evaluated from the difference between this image and subsequent images repeatedly taken after fixed number of shifts. In this experiment one image was taken after every 5 times of shifts and 10 images were captured in total. On each image the boundary of the circle was first manually marked by green dots (Fig. 4E), followed by a circle fitting to automatically detect the centroid. The repeatability error was $-1.2 \pm 0.5 \mu \mathrm{m}$ in $\mathrm{x}$ direction and $-0.1 \pm 0.3 \mu \mathrm{m}$ in $\mathrm{y}$ direction in mean \pm std format.

Position errors in $x$ and $y$ directions: A similar process was performed for the computation of position errors. The microscope was set to the working position. Instead of moving the microscope, the stage was translated within its plane to drive a circle in the image to the image center (Fig. 4D). The punch device was then shifted to the working position and make a hole on the paper. The hole position was recorded by the microscope when shifted back. Both the boundary of the black circle and the hole were manually marked (Fig. 4 $\mathrm{E}$ ). Circle fittings were performed to obtain both centroids and radii. The distance between the hole centroid and the image center were measured as the position errors. In order 


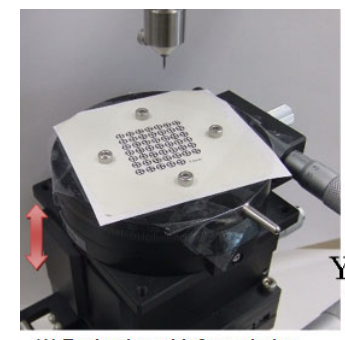

(A) Evaluation with $3 \mathrm{~mm}$ circles

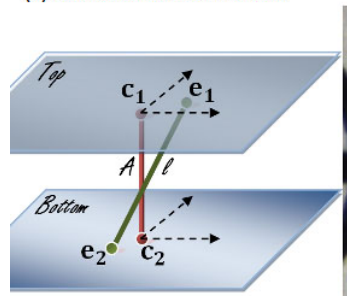

(B) Position and orientation errors

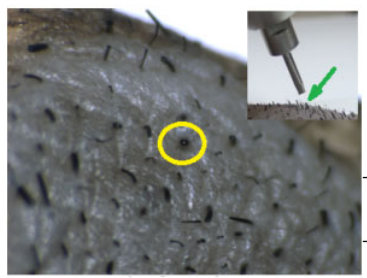

(C) In vitro experiment

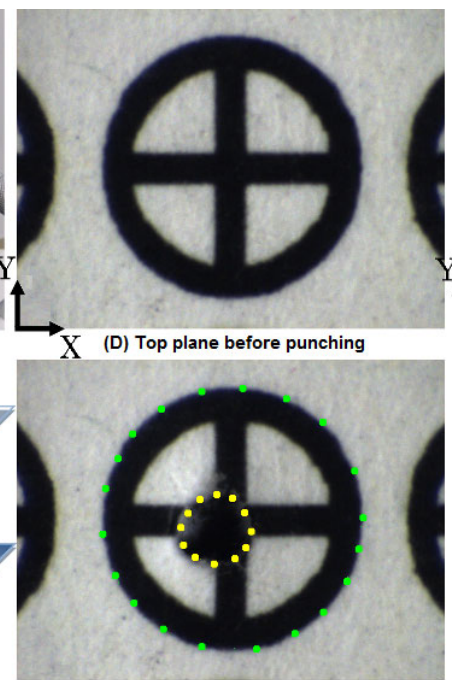

(E) Top plane after punching

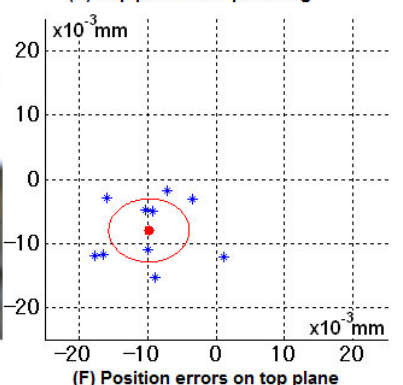

(F) Position errors on top plane

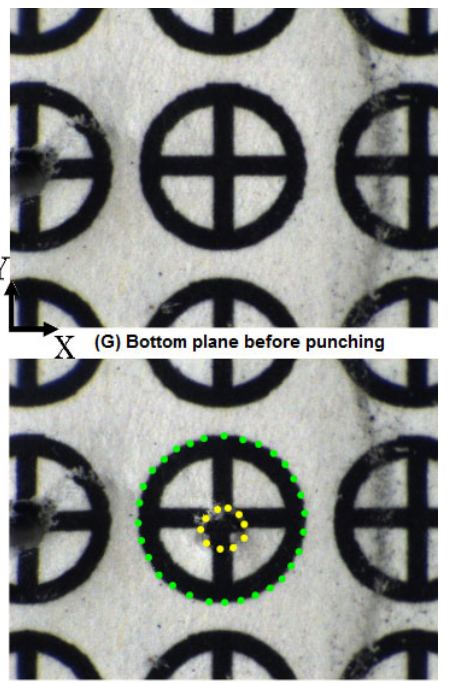

(H) Bottom plane after punching

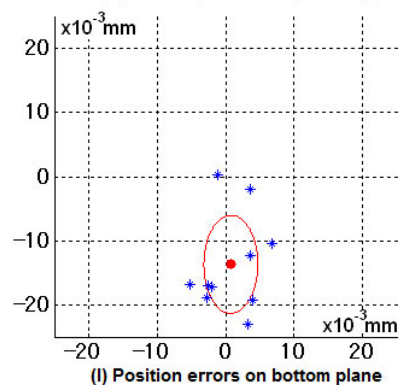

Fig. 4. Evaluation experiments, (A) setup, (B) in vitro experiment, (C) position errors and orientation errors, (D) and (E) images before and after punching on the top plane, $(\mathrm{F})$ position errors on the top plane, $(\mathrm{G})$ and $(\mathrm{H})$ images before and after punching on the bottom plane and (I) position errors on the bottom plane.

to cover the working space from the hair tip above the skin to the papillary bulb tip under the skin, a $6.0 \mathrm{~mm}$ thick area was defined for the evaluation. We set the top plane of the working space at the place $3.0 \mathrm{~mm}$ higher than the calibration plane and the bottom plane $3.0 \mathrm{~mm}$ lower than that plane. Fig. 4D-I show the results on the two planes. Different zoom magnification was applied on each plane (top plane: $38 \mathrm{x}$, bottom plane: $22 \mathrm{x}$ ), which were estimated from the pixel resolution of the $3.0 \mathrm{~mm}$ black circles. The resolution analysis was also used to convert the position errors from pixel to millimeter.

The test was performed 10 times on each plane. A consistent error of -9.8 $\pm 5.9 \mu \mathrm{m}$ in $\mathrm{x}$ direction and $-8.0 \pm 4.9 \mu \mathrm{m}$ in $\mathrm{y}$ direction on the top plane is observed. A slight large error in y direction which is $-13.7 \pm 7.6 \mu \mathrm{m}$ is found on the bottom plane. The error in $\mathrm{x}$ direction on that plane is $0.8 \pm 3.9 \mu \mathrm{m}$. The ellipses and their centers in Fig. 4F and $4 \mathrm{I}$ give the average errors and the standard deviations in $\mathrm{x}$ and $\mathrm{y}$ directions. 
Orientation error: The orientation error was determined from the average errors (the centers of the ellipse in Fig. 4F and 4I) on the top and the bottom planes. As illustrated in Fig. $4 \mathrm{~B}$, if the average error of the top plane is located at $e_{1}$ and the error of the bottom plane is at $e_{2}$, the angle between the line $l$ of $e_{1} e_{2}$ and the axis $A$ of the planes gives the orientation error. The direction of $A$ can be obtained by connecting two image centers, $c_{1}$ and $c_{2}$. The orientation error is 0.115 degrees from this calculation.

In vitro experiments were taken on the eyelid part of a 3 -month black porcine, which had hair similar to human scalp hair. A hair was first set to the image center (a black dot in Fig. 4C). It was observed that the whole hair could be inserted into the hollow needle with a right angle (small figure in Fig. 4C) after shifting. No hair was actually extracted because of the stiffness of porcine skin.

\section{Conclusion and Discussion}

In this paper, we proposed a new robotic system for hair harvesting process. A novel end-effector was developed which met the high accuracy requirement in this application. The results demonstrated that the proposed calibration method was efficient and reliable. The system is currently targeting straight hair patients only because of lack of information under the skin. Different imaging modalities, for example high frequency ultrasound or optical coherence tomography, had been tried but failed because of low image qualities. More image modalities will be considered in the future. Mechanical, electrical and software safeties are also needed to be investigated, e.g., force sensors for the needle insertion.

Acknowledgments. This work was partially supported by Grant-in-Aid for Scientific Research (21-09775) of the Japan Society for the Promotion of Science (JSPS). H. Liao was supported by Grant for Translational Systems Biology and Medicine Initiative from Ministry of Education, Culture, Sports, Science \& Technology of Japan, and JSPS Grant-in-Aid for Scientific Research (23680049).

\section{References}

1. International Society of Hair Restoration Surgery, 2009 RH.Research.: International Society of Hair Restoration Surgery: 2009 Practice Census Results (2009)

2. Bernstein, R., Rassman, W.: Follicular Transplantation. Dermatologic Surgery 23, 771-784 (1997)

3. Rassman, W., Bernstein, et al.: Follicular Unit Extraction: Minimally Invasive Surgery for Hair Transplantation. Dermatologic Surgery 28, 720-728 (2002)

4. Onda, M., Igawa, H., Inoue, K., Tanino, R.: Novel Technique of Follicular Unit Extraction Hair Transplantation with a Powered Punching Device. Dermatologic Surgery 24, 1683-1688 (2008)

5. Gildenberg, P.L.: Hair Transplantation Method and Apparatus. US Patent App. $10 / 354,836(2003)$

6. Bodduluri, M., Gildenberg, P.L.: Methods for Harvesting Follicular Units Using an Automated System. US Patent App. 11/380,903 (2006) 